\title{
Estilo de vida y glucemia capilar en personas con diabetes mellitus tipo 2
}

Lifestyle and capillary glycemia in persons with type 2 diabetes mellitus

Martínez Díaz Nazaria ${ }^{1 凶}$, Erika Mayte Del Ángel Salazar ${ }^{1}$, David Zepeta Hernández², Sandra Cuervo Zaragoza $^{3}$ y Blanca Estela Rivera Berman ${ }^{3}$

\begin{abstract}
${ }^{1}$ Docente de tiempo completo de la Facultad de Enfermería de la Universidad Veracruzana Región Poza RicaTuxpan, México. ${ }^{2}$ Docente por asignatura de la Facultad de Enfermería de la Universidad Veracruzana Región Rica-Tuxpan, México. ${ }^{3}$ Estudiante de la Facultad de Enfermería de la Universidad Veracruzana Región Poza Rica-Tuxpan, México.

\Autor para correspondencia: nmartinez@uv.mx
\end{abstract}

Recibido: 26/01/2016

Aceptado: 29/06/2016

\section{RESUMEN}

El estilo de vida es la manera general de vivir que se basa en la interacción entre las condiciones de vida y los patrones individuales de la conducta, los cuales están determinados por factores socioculturales y por las características personales de los individuos. La modificación de estos factores puede cambiar la historia natural de la diabetes mellitus e inclusive retrasar o evitar su aparición. El objetivo fue determinar los estilos de vida y nivel de glucemia capilar de personas con diabetes mellitus tipo 2 que acuden a un centro de salud. Estudio descriptivo, transversal y observacional. Se encuestó a 35 personas mediante el "Instrumento para Medir el Estilo de Vida en Diabéticos" (IMEVID) con un Alpha de Crombach de 0,81. Para determinar la glucosa capilar se utilizó el equipo Accu Chek Active. El estilo de vida tuvo una media de $62.4 \pm 9.55$, el $63 \%$ presentan un estilo de vida poco favorable. Los participantes no tienen un estilo de vida adecuado, pues los resultados evidencian predominio de estilos de vida poco favorables y desfavorables. En relación a la glucosa capilar, se encontró valores glucémicos elevados en la mayoría de las personas.

Palabras claves: Estilos de vida, diabetes mellitus tipo 2, glucosa capilar, enfermería.

\begin{abstract}
The lifestyle is the general way of living that is based on the interaction between living conditions and individual patterns of behavior, which are determined by sociocultural factors and personal characteristics of individuals. Changing these factors can change the natural history of diabetes mellitus
\end{abstract}


and even delay or prevent its occurrence. The objective was to determine lifestyles and level of blood glucose in persons with type 2 diabetes mellitus that attend to a health center. Descriptive, transversal and observational study. He polled 35 persons with the "Instrument to Measure Lifestyle in Diabetics" (IMEVID) with a Cronbach Alpha of 0.81. To determine capillary glucose Accu Chek Active equipment was used. The lifestyle has an average of $62.4 \pm 9.55,63 \%$ have a favorable little lifestyle. Participants do not have a right lifestyle, as the results demonstrate predominance of styles favorable little and unfavorable life. In relation to blood glucose levels, were found elevated glucose values in most persons.

Keywords: Lifestyle, tipe 2 diabetes mellitus, capillary glucose, nursing.

\section{INTRODUCCIÓN}

La enfermería es la ciencia encargada de la atención autónoma y en colaboración dispensada a personas de todas las edades, familias, grupos y comunidades, enfermos o no, $\mathrm{y}$ en todas circunstancias (Organización Mundial de la Salud, 2015). Se ocupa del cuidado de las respuesta humanas emanadas de patologías que afectan a la población, como las enfermedades crónicas degenerativas, entre ellas, la diabetes mellitus tipo 2 (DM2).

La OMS estima que en el mundo hay más de 347 millones de personas con diabetes, en el año 2012 fallecieron 1,5 millones de personas como consecuencias de altos niveles de azúcar en sangre. Se prevé que el número de personas con diabetes mellitus aumentará de 366 millones (2011) hasta 552 millones en 2030, es decir, un adulto de cada diez la padecerá. En México existen 6.4 millones de adultos con diabetes, es decir el $9.2 \%$ de la población adulta, esta cantidad podría elevarse al doble por aquellos diabéticos que aún no conocen su condición (Hernández-Ávila et al., 2013). El estado de Veracruz tiene una prevalencia de las más altas a nivel nacional, ocupando el tercer lugar, pues en el 2012 se reportaron 27,824 casos nuevos de diabetes (Servicios de Salud de Veracruz [SESVER], 2013).

Un aspecto relevante del control de todo padecimiento crónico degenerativo como la diabetes es que el paciente modifique los estilos de vida aprendidos. El estilo de vida es un constructo complejo que desempeña un papel central en el estado de salud enfermedad. Algunos de sus componentes (tipo de alimentación, actividad física, consumo de tabaco, ingesta de alcohol y estrés crónico entre otros) se han asociado a la aparición, el curso clínico y control de la DM2 y sus complicaciones. La modificación de estos factores puede cambiar la historia natural de esta enfermedad e inclusive retrasar o evitar su aparición (López-Carmona et al., 2004).

La OMS define al estilo de vida como la manera general de vivir que se basa en la interacción entre las condiciones de vida y los patrones individuales de la conducta, los cuales están determinados por factores socioculturales y por las características personales de los individuos (García-Laguna et al., 2012).

\section{Revista Científica Biológico Agropecuaria Tuxpan 4 (1)}


El tratamiento nutricional es una de las piedras angulares para lograr los objetivos terapéuticos, todos los profesionales de la salud que tratan pacientes con diabetes saben lo difícil que es lograr un adecuado control glucémico en el paciente que tiene malos hábitos de alimentación. Los alimentos deben racionarse durante el día para que el azúcar en la sangre tenga siempre las mismas cifras. Se sugiere ingerir 3 comidas principales y 2 colaciones una a media mañana y otra a media tarde. Reducir la cantidad de sal que se utiliza en la preparación de los alimentos.

También la actividad física regular se asocia a una reducción en el riesgo de morbilidad y mortalidad cardiovascular (Minsal, 2010). Otro estilo no saludable que debe evitarse es el alcohol. Este puede ocasionar hipoglucemia ya que potencia la acción de la insulina y disminuye la respuesta hepática a la disminución de la glucemia (Cruz, 2009).

Diversos estudios han demostrado una mayor prevalencia de tabaquismo en pacientes diabéticos ajustado por edad, sexo, raza y nivel educativo. La suspensión del tabaquismo es una herramienta terapéutica que ha mostrado mayor beneficio en el paciente con diabetes (Huerta, 2006).

Habría que decir también que el aspecto psicológico es de suma importancia, puesto que las personas con diabetes sufren depresión, este factor puede influir en la capacidad de las personas para manejar su enfermedad. Ante este panorama, el objetivo del presente estudio fue identificar los estilos de vida, incluyendo el tipo de alimentación, el tiempo que dedican las personas a realizar ejercicio, la presencia de toxicomanías, la situación emocional y la adherencia al tratamiento así como el nivel de glucemia de las personas con Diabetes Mellitus tipo II, adscritas a un centro de salud.

\section{MATERIALES Y MÉTODOS}

Estudio descriptivo, transversal y observacional, se realizó un muestreo por conveniencia de 35 personas con DM2 que acuden a un centro de salud del Municipio de Martínez de la Torre, Veracruz. Las variables de estudio fueron los estilos de vida y la glucemia capilar a través de una encuesta donde se aplicó el cuestionario denominado "Instrumento para Medir el Estilo de Vida en Diabéticos" (IMEVID) con un Alpha de Crombach de 0,81, elaborado por LópezCarmona et al. en 2003 para conocer y medir el estilo de vida en los pacientes con DM2 de una manera rápida y fácil. El instrumento está constituido por 25 Ítems cerrados agrupadas en 7 dominios: nutrición, actividad física, consumo de tabaco, consumo de alcohol, información sobre diabetes, manejo de emociones y cumplimiento del tratamiento. Cada ítem presenta tres opciones de respuesta con calificaciones de 0,2 y 4, donde 4 corresponde al valor máximo deseable en cada respuesta, para una puntuación total de 0 a 100, sin valores nones en la escala. El instrumento clasifica a los sujetos de la siguiente manera: una puntuación $<60$ corresponde a un estilo de vida desfavorable, entre 60 y 80, a un estilo de vida poco favorable, y más de 80 puntos, a un estilo de vida favorable. Para determinar la glucosa capilar preprandrial, se utilizó el equipo Accu Chek Active tomando como valores de referencia de 70 a $130 \mathrm{mg} / \mathrm{dl}$ como normo glucemia y >131 mg/dl como hiperglucemia capilar.

El cuestionario fue entregado a cada uno de los participantes de manera voluntaria y anónima. La aplicación del cuestionario fue en forma personal, autoaplicado y sin control de tiempo. Una vez recolectados los cuestionarios debidamente requisitados, se realizó la revisión y codificación de los mismos, en seguida se elaboró el diseño y la captura de la base de 
datos para poder llevar a cabo la validación y análisis, con apoyo de Excel y SPSS versión 19.0.

Dicha investigación se realizó en apego a las normas éticas internacionales para investigaciones en seres humanos y sustentada en el reglamento de la Ley General de Salud conforme a lo mencionado en los artículos 100 y 101 la cual menciona que toda investigación realizada con seres humanos deberá adaptarse a los principios científicos y éticos que justifiquen la investigación médica, y solo podrá realizarse cuando exista la seguridad de no exponer riesgos ni daños al sujeto a la experimentación, pues se considera una investigación sin riesgo debido a que se emplearon técnicas y métodos de investigación documental. Así mismo, se contó con la firma del consentimiento informado por los participantes (Artículo 17 del reglamento de la Ley General de Salud).

\section{RESULTADOS}

Se encuestó a 35 personas con diabetes mellitus tipo 2 con una edad promedio de 56.2 \pm 10.4 años, una edad mínima de 38 y máxima de 78 años. Con respecto al género, el 69\% fueron mujeres y el $31 \%$ hombres. En relación a la escolaridad, el 3\% tiene licenciatura y preparatoria, el $14 \%$ secundaria, el $46 \%$ primaria y $34 \%$ es analfabeta.

En cuanto al análisis de la glucosa capilar, se obtuvo una media de 174. $29 \pm 69.2$ $\mathrm{mg} / \mathrm{dl}$, con un valor mínimo de $76 \mathrm{mg} / \mathrm{dl}$ y un máximo $401 \mathrm{mg} / \mathrm{dl}$. Al comparar los niveles de glucosa con los valores de referencia, se encontró que el $34 \%$ de los participantes obtuvieron un nivel de glucosa normal, mientras que el $66 \%$ presentaron un nivel de glucosa elevada (Cuadro I).

\begin{tabular}{ccc} 
Cuadro I. Glucosa capilar preprandrial \\
\hline Glucosa & Frecuencia & Porcentaje \\
\hline $70-130 \mathrm{mg} / \mathrm{dl}$ & 12 & 34 \\
$>131 \mathrm{mg} / \mathrm{dl}$ & 23 & 66 \\
\hline
\end{tabular}

Respecto al análisis del estilo de vida, en el puntaje total se obtuvo una media de $62.4 \pm$ 9.55. Se encontró que el $37 \%$ de los participantes presentan un estilo de vida desfavorable, mientras que el $63 \%$ manifestó un estilo de vida poco favorable (Cuadro II). 
Cuadro II. Tipos estilo de vida en personas con DM2

\begin{tabular}{ccc}
\hline Estilos de vida & Frecuencia & Porcentaje \\
\hline Poco adecuado & 13 & 37 \\
Desfavorable & 22 & 63 \\
\hline
\end{tabular}

\section{DISCUSIÓN}

En el presente estudio se encontró un predomino del sexo femenino con el 69\%, semejante al estudio de Cantú (2014) y Figueroa-Suarez et al. (2014) e igual al estudio de López-Carmona et al. (2004) con un 72\%, $73 \%$ y $69 \%$ respectivamente. En relación a la edad, se obtuvo un promedio de $56.2 \pm 10.4$ años, similar a los resultados de LópezCarmona et al. (2004) con un valor de $54.8 \pm$ 10.8 y Figueroa-Suarez et al. (2014) con un media de $56.51 \pm 9.62$ años.

Respecto al nivel educativo, en el presente estudio se encontró que la mayoría tiene estudios de primaria con un $46 \%$, resultados parecidos al estudio de Figueroa-Suarez et al. (2014) con un 47\%, y diferente a lo que reportó Cantú en 2014 donde el $78 \%$ de los participantes tenían estudios de nivel primaria.

En relación a los niveles de glucosa capilar, se obtuvo una media $174.29 \pm 69.2$ $\mathrm{mg} / \mathrm{dl}$, similar a lo encontrado por FigueroaSuarez et al. (2014) con un media de $149.21 \pm$ $49.34 \mathrm{mg} / \mathrm{dl}$. En cuanto a la valoración del estilo de vida, se obtuvo una media de 62.4 semejante a lo reportado por López-Carmona et al. (2004) con promedio de 68, y FigueroaSuarez et al. (2014) con un puntaje promedio de 73. Los resultados muestran que el $63 \%$ de los participantes tienen un estilo de vida poco favorable $y$ el $37 \%$ un estilo de vida desfavorable, resultados diferentes a los de Cantú (2014).

\section{CONCLUSIONES}

Respecto a las variables de estudio, se determina que los participantes no tienen un estilo de vida favorable, pues los resultados evidencian predominio de estilos de vida poco favorables y desfavorables; una dieta poco saludable, actividad física insuficiente, falta de sistematización en la toma de sus medicamentos, consumo de tabaco y malas condiciones en su estado emocional. En relación a la glucosa capilar, se encontró valores glucémicos elevados en la mayoría de los participantes, resultado congruente con el estilo de vida que manifestaron.

\section{LITERATURA CITADA}

Cantú, P. (2014). Estilo de vida en pacientes adultos con diabetes Mellitus tipo 2. Rev. Enfermería actual en costa Rica. 1(27), 1-14.

Cruz E, Pino J (2009) Estilo de vida relacionado con la salud. Universidad de Murcia 5(1) 20-40.

\section{Revista Científica Biológico Agropecuaria Tuxpan 4 (1)}

ISSN: 2007-6940 
Figueroa, M, Cruz, E, (2014). Estilo de vida y control metabólico en Diabéticos del programa DiabetIMSS. Gaceta medica de México. 1(1) 30ᄀ33

García-Laguna, D.G., García-Salamanca, G.P., Tapiero-Paipa, Y.T. y Ramos, C.D.M. (2012). Determinantes de los estilos de vida y su implicación en la salud de jóvenes universitarios. Revista Hacia la Promoción de la Salud; 17 (2)169-185.

Hernández-Ávila, M., Gutiérrez, J.P., ReynosoNoverón, N. (2013). Diabetes mellitus en México. El estado de la epidemia. Salud Pública de México: 55 (2) 129136. https://doi.org/10.21149/spm.v55s2.5

López-Carmona, J.M., Ariza-Andraca, C.R., Rodríguez-Moctezuma, J.R. y Munguía, M.C. (2003). Construcción y validación inicial de un instrumento para medir el estilo de vida en pacientes con diabetes mellitus tipo 2. Salud pública Méx .45

(4) $8-12$ https://doi.org/10.1590/S0036-36342003 López-Carmona, J.M., Rodríguez-Moctezuma, J.R., Ariza-Andraca, C.R. y MartinezBermudez, M. (2004). Estilo de vida y control metabólico en pacientes con diabetes mellitus tipo 2. Validacion por constructo del IMEVID. Atención Primaria; 33 (1) 20-7. https://doi.org/10.1016/S0212-6567(04)7

Minsal, S (2010). Ministerio de salud. Guía Clínica Diabetes Mellitus tipo 2 Santiago chile. 1(1) 2-74.

Organización Mundial de la Salud. Temas de salud. Enfermería. Información consultada el 29 de febrero de 2015 en: http://www.who.int/topics/nursing/es/
Servicios de Salud de Veracruz. (2013) Programa de acción específico: diabetes mellitus. Información consultada el 29 de febrero en http://web.ssaver.gob.mx/cronicasnotran smisibles/files/2013/10/ programa-anual-de-diabetes-mellitus2013.pdf 


\title{
Copyright (c) 2016 Nazaria Martinez Diaz, Erika Mayte Del Ángel Salazar, David Zepeta Hernández, Sandra Cuervo Zaragoza y Blanca Estela Rivera Berman
}

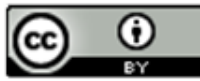

Este tex to está protegido por una licencia licencia Creative Commons $\underline{4.0}$

\begin{abstract}
Usted es libre para Compartir — copiar y redistribuir el material en cualquier medio o form ato- y Adaptar el documento - remezclar, transformar y crear a partir del material- para cualquier propósito,, incluso para fínes comerciales, siempre que cumpla la condición de:

Atribución: Usted debe dar crédito a la obra original de manera adecuada, proporcionar un enlace a la licencia, e in dicar si se han realizado cam bios. Puede hacerlo en cualquier form a razonable, pero no de form a tal que sugiera que tiene el apoyo del licenciante o lo recibe por el uso que hace de la obra.

Resumendelicencia - Textocompletodelalicencia
\end{abstract}

